\section{Custo-efetividade de um programa de rastreamento organizado de câncer de mama no Sul do Brasil}

\author{
Cost-effectiveness of an organized breast cancer \\ screening program in Southern Brazil
}

\author{
Coste-efectividad de un programa organizado \\ para la detección precoz del cáncer de mama \\ en el sur de Brasil
}

Rodrigo Antonini Ribeiro ${ }^{1}$

Maira Caleffi 1

Carisi Anne Polanczyk 1

\footnotetext{
1 Instituto de Educação e Pesquisa, Hospital Moinhos de Vento, Porto Alegre, Brasil.

Correspondência R. A. Ribeiro Instituto de Educação e Pesquisa, Hospital Moinhos de Vento.

Rua Ramiro Barcelos 910 Porto Alegre, RS 90630-070, Brasil.

rodrigo.ribeiro@hmv.org.br
}

\begin{abstract}
The aim of this study was to evaluate the costeffectiveness of an organized breast cancer mammographic screening program implemented in Porto Alegre (Núcleo Mama Porto AlegreNMPOA), Rio Grande do Sul State, Brazil. A Markov model was constructed to estimate the incremental cost-effectiveness ratio of NMPOA compared to current BC diagnosis and care in the Brazilian public health system, in a hypothetical cohort of women aged 40-69 years at risk of developing breast cancer. Model parameters were collected from NMPOA and the national literature. In the NMPOA strategy, effectiveness was modeled taking into account the actual observed screening adherence. Effectiveness was measured in quality-adjusted life years (QALYs). Incremental cost-effectiveness ratio in the base case was $R \$ 13,426$ per QALY. This result was not sensitive to variation in the main model parameters in sensitivity analyses. Considering the threshold usually suggested as highly attractive in Brazil, breast cancer screening as performed in NMPOA is cost-effective in cities with high incidence of breast cancer.
\end{abstract}

Breast Neoplasms; Mass Screening; Cost-Effectiveness; Health Technology Evaluation

\section{Resumo}

O objetivo deste estudo foi avaliar o custo-efetividade de um programa organizado de rastreamento mamográfico de câncer de mama implementado na cidade de Porto Alegre, Rio Grande do Sul, Brasil (Núcleo Mama Porto Alegre NMPOA). Foi construído modelo de Markov para estimar a relação de custo-efetividade incremental do NMPOA em comparação à situação atual de atenção ao câncer de mama no SUS, em coorte hipotética de mulheres de 40-69 anos com risco de desenvolver câncer de mama. Os parâmetros foram coletados do NMPOA e da literatura nacional. Na estratégia NMPOA, a efetividade foi modelada levando-se em conta a real adesão da população participante do rastreamento. A efetividade foi medida em anos de vida ajustados para qualidade (QALY). A relação de custoefetividade incremental no caso base foi de $R \$$ 13.426,00 por QALY. Esse resultado foi pouco sensível à variação dos principais parâmetros do modelo nas análises de sensibilidade. Considerando o limiar usualmente sugerido como bastante atrativo do ponto de vista econômico no Brasil, o rastreamento do câncer de mama nos moldes do NMPOA é custo-efetivo em cidades com alta incidência deste tipo de câncer.

Neoplasias da Mama; Programas de Rastreamento; Custo Efetividade; Avaliação de Tecnologias de Saúde 


\section{Introdução}

O câncer de mama é a principal causa de morte por neoplasia em mulheres no Brasil, tendo o risco estimado de 52 casos por 100 mil mulheres 1 . Essas estimativas colocam o câncer de mama como grande problema de saúde pública, não só pelo impacto em morbimortalidade, mas também pelos custos associados à doença e seu tratamento 2 .

Tendo em vista que não são conhecidas as maneiras eficazes de reduzir a incidência da doença, o rastreamento do câncer de mama, cujo principal objetivo é diagnosticar a enfermidade em estágios iniciais, é uma das principais medidas disponíveis para diminuição dessa morbimortalidade ${ }^{3}$. Nesse contexto, a mamografia - principal método para rastreio - mostrou, em metanálise de ensaios clínicos randomizados, redução de ao menos $15 \%$ na mortalidade pela doença ${ }^{4}$. No Brasil, está prevista, desde 2004, a mamografia de rastreamento pelo menos a cada dois anos nas mulheres entre 50-69 anos e anualmente a partir dos 35 para as mulheres com risco elevado 5 , sendo que mais recentemente foi expandida a mamografia para mulheres a partir de 40 anos de idade (por meio da Lei no 11.664), sem definição clara da sua inserção na política vigente. Apesar da existência dessas políticas, não existe um programa nacional de rastreamento organizado efetivamente implementado.

Tendo em vista o custo significativo da implementação de programas de rastreamento mamográfico, vários países avaliaram a custoefetividade dos mesmos, encontrando resultados heterogêneos dependendo dos pressupostos e parâmetros incluídos nos modelos econômicos 6,7,8. No Brasil, apenas um estudo publicado até agora avaliou o custo-efetividade do rastreamento mamográfico, porém comparando cenários de $100 \%$ e $0 \%$ de adesão ao rastreamento ${ }^{9}$ - ambos pouco plausíveis. A relação de custo-efetividade incremental encontrada foi de $\mathrm{R} \$ 13.573,00$ por ano de vida ganho.

No ano de 2004, foi iniciado o projeto Núcleo Mama Porto Alegre (NMPOA), Rio Grande do Sul, Brasil, resultado de uma parceria público-privada entre a Associação Hospitalar Moinhos de Vento e a Secretaria Municipal da Saúde de Porto Alegre, contando com o apoio do Ministério da Saúde a partir de 2009 10. A principal meta do projeto é avaliar a efetividade de um programa organizado de rastreamento de câncer de mama para mulheres em situação de vulnerabilidade social. Os dados desse projeto criam uma oportunidade ímpar de avaliação econômica do rastreamento do câncer de mama no Brasil, uma vez que refletem o funcionamento de um pro- grama efetivamente implementado em cenário nacional.

O objetivo deste estudo foi estimar a custoefetividade de um programa de rastreamento de câncer de mama nos moldes do NMPOA, avaliando o ganho em expectativa de vida (e a mesma ajustada para qualidade) e a diferença nos custos, na comparação com a situação atual do rastreamento e tratamento do câncer de mama em mulheres usuárias da rede pública de saúde.

\section{Métodos \\ População alvo}

A população alvo do modelo é uma coorte hipotética de mulheres que inicia o seguimento sem câncer de mama, com idades entre 40 e 69 anos, tendo risco de desenvolvimento desta doença ao longo do tempo. O caso base refletirá uma população com alta incidência de câncer de mama na população feminina em geral, como Porto Alegre (incidência de 126 casos por 100 mil mulheres em 2010); porém, será avaliada a incidência mais baixa nas análises de sensibilidade, para refletir a epidemiologia de outras regiões do Brasil. Para garantir a validade interna do modelo, a distribuição da faixa etária das mulheres na entrada no modelo seguirá aquela observada em Porto Alegre.

\section{Estratégias em comparação e estrutura do modelo}

O modelo compara duas abordagens para o diagnóstico precoce do câncer de mama. A primeira é baseada no modelo do NMPOA (descrito em detalhes em publicação prévia 10), cujas principais características são: (1) rastreamento anual, com mamografia e exame clínico das mamas; (2) controle centralizado da frequência de rastreamento das participantes, com busca ativa de mulheres que não estão cumprindo os intervalos regulares entre as vindas ao programa; (3) realização de todas as etapas do cuidado - desde o rastreamento até a cirurgia para retirada do tumor mamário - no mesmo centro, com o intuito de agilizar o processo.

A segunda estratégia, doravante chamada de cuidado usual, reflete a situação atual de rastreamento e tratamento do câncer de mama nas pacientes usuárias da rede pública de saúde. Ainda que exista uma política vigente proposta pelo Instituto Nacional de Câncer (INCA) de que o rastreamento mamográfico seja feito bianualmente (em mulheres entre 50-69 anos) e o exame clínico anualmente (entre 40-69 anos) 5, a inexistên- 
cia de coordenação do fluxo nos moldes de um programa de rastreamento organizado acaba por configurar esta estratégia como uma combinação de rastreamento oportunista para algumas mulheres que procuram o sistema por iniciativa própria, e ausência de rastreamento para as que não fazem tal procura.

O modelo empregado para comparar as estratégias foi o de Markov. Em ambas, o modelo inicia com $100 \%$ da população sem câncer de mama, havendo surgimento da doença de acordo com sua incidência. Os casos incidentes são distribuídos em estadiamentos (I ao IV). Cada estadiamento tem uma taxa de sobrevida prevista (de acordo com a busca de parâmetros na literatura, conforme detalhado a seguir), e não difere nas duas estratégias: uma paciente com estadiamento I na estratégia NMPOA tem a mesma sobrevida que uma paciente no mesmo estágio no cuidado usual. Portanto, o benefício da intervenção NMPOA se dá por meio de uma proporção maior de pacientes em estágios precoces, na comparação com o cuidado usual. A representação esquemática do modelo pode ser vista na Figura 1.

\section{Pressupostos do modelo}

Foram adotados os seguintes pressupostos:

- A perspectiva foi do Sistema Único de Saúde (SUS).

- O horizonte temporal foi de tempo de vida.

- A taxa de desconto foi de $5 \%$ ao ano para custo e efetividade.

- A efetividade foi mensurada em anos de vida ajustados para qualidade (QALY - quality adjusted life years).

- Os anos de vida acumulados por mulheres sem câncer, em ambas as estratégias, foram calculados de acordo com as tábuas de vida do Instituto Brasileiro de Geografia e Estatística (IBGE). - Os anos de vida acumulados por mulheres com câncer foram ditados pelo estadiamento no momento do diagnóstico, sendo baseados em estudos de coorte nacionais. A relação entre estadiamentos e prognóstico é muito bem estabelecida na literatura 11, e também existem dados nacionais com boa qualidade fazendo esta correlação em cenário local 12,13. Portanto, a efetividade de cada estratégia é resultado dos estadiamentos obtidos.

- Os estadiamentos dos casos de câncer diagnosticados no cuidado usual são baseados em estudos observacionais nacionais, de forma a refletir a realidade local 12,13,14,15,16.

- Os estadiamentos dos casos de câncer diagnosticados no NMPOA foram estimados com base na frequência da realização de mamogra- fias pela população cadastrada no NMPOA. Não foram utilizados os dados de casos efetivamente detectados, tendo em vista a pequena casuística acumulada até o presente e consequente imprecisão das estimativas. Optamos por quantificar a frequência de realização de mamografias e correlacionar tal parâmetro com os estadiamentos tumorais esperados, uma vez que dados consistentes da literatura referendam a validade deste parâmetro intermediário 17 .

- As possíveis complicações do câncer de mama foram baseadas em dados nacionais, impactando basicamente em custos. Como casos com estadiamentos mais avançados tendem a sofrer mais internações hospitalares, o custo de seguimento destes é maior.

\section{Estimativas dos parâmetros para avaliação} da efetividade das estratégias

Para a incidência de câncer de mama do caso base foram considerados os dados de Porto Alegre, cidade sede do NMPOA e com alta incidência deste tipo de câncer. Foi consultado o Registro de Câncer de Base Populacional 18, a fim de obterem-se dados estratificados por idade, que são mostrados na Tabela 1.

Conforme comentado anteriormente, a frequência de realização da mamografia é o principal dado do NMPOA para estimar a efetividade do programa, por meio do impacto da assiduidade das participantes nos estadiamentos tumorais, e por consequência, na sobrevida. Foram utilizadas as datas de realização das mamografias para estimar a frequência de realização dos exames. As mulheres foram classificadas em: (1) sem rastreamento; (2) rastreamento anual; (3) rastreamento bianual; (4) rastreamento trianual; (5) rastreamento irregular.

$\mathrm{Na}$ medida em que muitas mulheres não mantinham um intervalo de tempo regular entre as diversas vindas ao programa, alguns critérios foram adotados para a análise dos dados:

- Mulheres que estavam cadastradas no programa NMPOA há pelo menos 12 meses, porém nunca haviam comparecido, foram classificadas como "sem rastreamento".

- Mulheres que haviam comparecido pela última vez há mais de 40 meses foram consideradas como "rastreamento irregular", assim como as que tivessem tido algum intervalo entre mamografias maior que 40 meses.

- Mulheres que vieram apenas uma vez e cuja vinda tinha ocorrido entre 12 e 18 meses antes do fechamento da análise foram classificadas como "rastreamento anual"; entre 18 e 30 meses, "rastreamento bianual"; e entre 30 e 40 , "rastreamento trianual". 


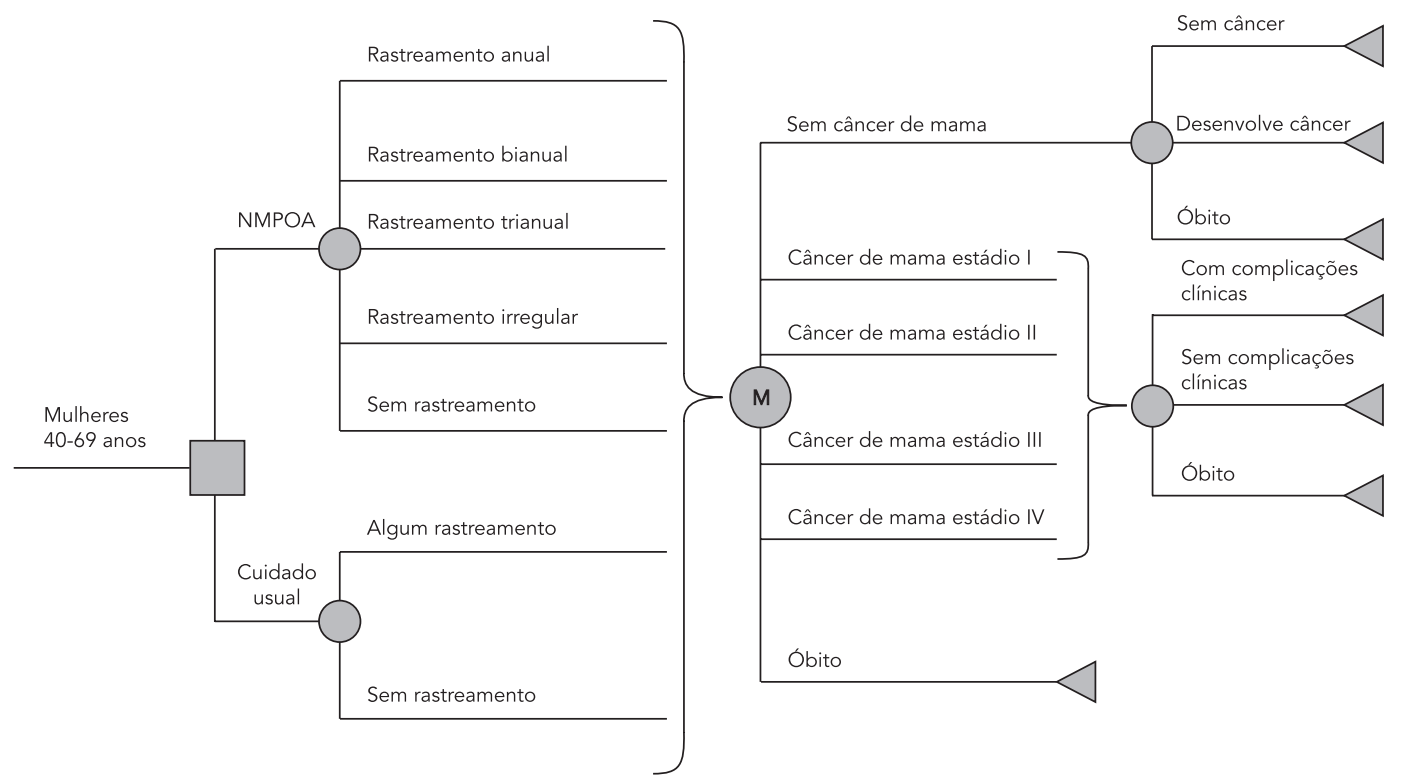

NMPOA: Núcleo Mama Porto Alegre.

- Para as demais mulheres, foram avaliados os intervalos entre as mamografias realizadas, sendo calculado o intervalo médio e o desvio-padrão destes tempos. Mulheres que tinham um desviopadrão menor do que seis meses foram classificadas de acordo com o tempo médio entre suas mamografias: 18 meses ou menos como rastreamento anual; entre 18 e 30 meses, rastreamento bianual; mais do que 30 meses, rastreamento trianual. Nas mulheres cujo desvio-padrão foi de seis meses, contou-se o número de intervalos entre 12 e 18 meses, entre 18 e 30, e entre 30 e 40 meses. Após, verificou-se qual o intervalo foi mais frequente, e o mesmo foi tido como o padrão preponderante de rastreamento da pessoa analisada.

O resultado final dessa análise pode ser visto na Tabela 1. Depois de estabelecer a frequência de rastreamento, procedemos a associação desta com os estadiamentos tumorais. A relação entre as frequências anual e bianual de rastreamento e os consequentes estadiamentos foram obtidos em uma publicação americana que analisou cerca de oito mil casos de câncer de mama, com análise retrospectiva do padrão de frequência de rastreamento 17 . Um ensaio clínico randomizado proveu dados para o rastrea- mento trianual 19. Para a estimativa dos estadiamentos de mulheres que faziam rastreamento irregular, foram utilizados os estadiamentos encontrados nos estudos nacionais que avaliaram a população usuária do SUS (descritos adiante). Apesar de saber-se que uma parcela dessa população realiza rastreamento regularmente, consideramos que esta seja pequena. Dados dos estadiamentos em pacientes que não faziam rastreamento não foram encontrados na literatura nacional; nossa abordagem foi utilizar as mesmas proporções de estadiamentos encontradas no rastreamento irregular.

Para o parâmetro de proporção de estadiamentos no cuidado usual, foram encontrados estudos de diferentes regiões do Brasil, incluindo Rio Grande do Sul 16, Santa Catarina 12, Minas Gerais 13, Rio de Janeiro 15 e um trabalho do INCA abrangendo várias regiões do país 14 . Todos eles apresentavam a proporção de estadiamentos no momento do diagnóstico. Utilizando os dados desses artigos, foi calculada a média ponderada da proporção de cada estadiamento (Tabela 1). É importante citar que o estudo do INCA domina os resultados, visto que sua amostragem é muito maior que a dos demais trabalhos. 
Tabela 1

Parâmetros do modelo.

\begin{tabular}{|c|c|c|}
\hline Parâmetros & Valores & Referências \\
\hline \multicolumn{3}{|l|}{ Parâmetros para efetividade } \\
\hline Anual & & White et al. 17 \\
\hline I & $70,40 \%$ & \\
\hline II & $26,10 \%$ & \\
\hline III & $1,75 \%$ & \\
\hline IV & $1,75 \%$ & \\
\hline Bianual & & White et al. 17 \\
\hline । & $68,00 \%$ & \\
\hline II & $27,10 \%$ & \\
\hline III & $2,45 \%$ & \\
\hline Trianual & & Breast Screening Frequency Trial Group 19 \\
\hline । & $62,00 \%$ & \\
\hline II & $29,00 \%$ & \\
\hline III & $4,50 \%$ & \\
\hline IV & $4,50 \%$ & \\
\hline \multirow[t]{2}{*}{ Irregular/Sem rastreamento/Situação atual do rastreamento } & & Schneider et al. 12; Guerra et al. 13; Thuler \& \\
\hline & & Mendonça 14; Brito et al. 14; Moraes et al. 16 \\
\hline I & $12,56 \%$ & \\
\hline II & $43,07 \%$ & \\
\hline III & $32,27 \%$ & \\
\hline IV & $12,10 \%$ & \\
\hline Mortalidade anual por câncer de mama de acordo com o & & Schneider et al. 12; Guerra et al. 13 \\
\hline II & $2,52 \%$ & \\
\hline III & $8,30 \%$ & \\
\hline IV & $22,87 \%$ & \\
\hline Probabilidade de internações por ano & & Dados primários HCPA e ICESP \\
\hline I & $11,00 \%$ & \\
\hline II & $32,00 \%$ & \\
\hline III & $62,00 \%$ & \\
\hline IV & $27,00 \%$ & \\
\hline Frequência de rastreamento no NMPOA & & Dados primários NMPOA \\
\hline Anual & $53,00 \%$ & \\
\hline Bianual & $12,50 \%$ & \\
\hline Trianual & $2,90 \%$ & \\
\hline Irregular & $23,10 \%$ & \\
\hline Nunca & $8,50 \%$ & \\
\hline $\begin{array}{l}\text { Taxa de realização de ecografia NMPOA (\% das mamografias desta } \\
\text { forma complementadas) }\end{array}$ & $23,00 \%$ & Dados primários NMPOA \\
\hline $\begin{array}{l}\text { Percentual anual de utilização de mamografia na situação atual de } \\
\text { rastreamento }\end{array}$ & $14,00 \%$ & Marchi et al. 27 \\
\hline
\end{tabular}

(continua) 
Tabela 1 (continuação)

\begin{tabular}{|c|c|c|}
\hline Parâmetros & Valores & Referências \\
\hline \multicolumn{3}{|l|}{ Parâmetros para efetividade } \\
\hline Incidência de câncer de mama em Porto Alegre por faixa etária (anos) & & INCA 18 \\
\hline \multicolumn{3}{|l|}{ (casos/100 mil mulheres) } \\
\hline $40-44$ & 134 & \\
\hline $45-49$ & 207 & \\
\hline $50-54$ & 252 & \\
\hline $55-59$ & 317 & \\
\hline $60-64$ & 323 & \\
\hline $65-69$ & 366 & \\
\hline $70-74$ & 366 & \\
\hline $75-79$ & 443 & \\
\hline $80-84$ & 451 & \\
\hline$>85$ & 400 & \\
\hline \multicolumn{3}{|l|}{ Parâmetros para custos } \\
\hline \multicolumn{3}{|l|}{ Tipo de cirurgia oncológica } \\
\hline Estádio 0/I & & $\begin{array}{c}\text { Dados não publicados Núcleo Mama Moinhos, } \\
\text { NMPOA e HCPA/ICESP }\end{array}$ \\
\hline Setorectomia & $73,00 \%$ & \\
\hline Mastectomia & $27,00 \%$ & \\
\hline Estádio ॥ & & $\begin{array}{l}\text { Dados não publicados Núcleo Mama Moinhos, } \\
\text { NMPOA e HCPA/ICESP }\end{array}$ \\
\hline Setorectomia & $39,00 \%$ & \\
\hline Mastectomia & $61,00 \%$ & \\
\hline Estádio III & & $\begin{array}{l}\text { Dados não publicados Núcleo Mama Moinhos, } \\
\text { NMPOA e HCPA/ICESP }\end{array}$ \\
\hline Setorectomia & $18,00 \%$ & \\
\hline Mastectomia & $73,00 \%$ & \\
\hline Nenhuma & $9,00 \%$ & \\
\hline Estádio IV & & Estimativa \\
\hline Mastectomia & $70,00 \%$ & \\
\hline Nenhuma & $30,00 \%$ & \\
\hline \multicolumn{3}{|l|}{ Realização de cirurgia de reconstrução } \\
\hline Pacientes abaixo de 70 anos & $70,00 \%$ & Estimativa \\
\hline Pacientes acima de 70 anos & $0,00 \%$ & \\
\hline \multicolumn{3}{|l|}{ Utilização de bloqueio hormonal por cinco anos } \\
\hline Tamoxifeno & $64,00 \%$ & Dados Núcleo Mama Moinhos e estimativa \\
\hline Anastrazol ou letrozol & $16,00 \%$ & \\
\hline \multicolumn{3}{|l|}{ Utilização de quimioterapia (adjuvante e neoadjuvante) } \\
\hline Estádio I & $39,00 \%$ & $\begin{array}{c}\text { Dados não publicados Núcleo Mama Moinhos, } \\
\text { NMPOA e HCPA/ICESP }\end{array}$ \\
\hline Estádios II a IV & $100,00 \%$ & Estimativa \\
\hline Fração de pacientes que fizeram quimioterapia em neoadjuvância & & Dados não publicados Núcleo Mama Moinhos \\
\hline Estádio I & $19,80 \%$ & \\
\hline Estádio II & $19,90 \%$ & \\
\hline Estádio III & $23,00 \%$ & \\
\hline Utilização de radioterapia (30 ciclos) & & Estimativa \\
\hline Pacientes submetidas à setorectomia & $100,00 \%$ & \\
\hline Pacientes estadiamento III & $100,00 \%$ & \\
\hline Pacientes estadiamento IV & $50,00 \%$ & \\
\hline
\end{tabular}

(continua) 
Tabela 1 (continuação)

\begin{tabular}{|c|c|c|}
\hline Parâmetros & Valores & Referências \\
\hline \multicolumn{3}{|l|}{ Parâmetros para custos } \\
\hline Número de procedimentos na população para cada caso de câncer & & Dados primários NMPOA \\
\hline Agulhamento & 0,67 & \\
\hline Biópsia de fragmento & 2,09 & \\
\hline Punção aspirativa & 1,91 & \\
\hline \multicolumn{3}{|l|}{ Valores de insumos } \\
\hline Exames & & Tabelas de remuneração do SUS \\
\hline Mamografia de rastreamento ou diagnóstica & $R \$ 45,00$ & \\
\hline Ecografia mamária & $\mathrm{R} \$ 24,20$ & \\
\hline Biópsia por fragmento (incluindo anatomopatológico) & $\mathrm{R} \$ 92,43$ & \\
\hline Punção aspirativa (incluindo anatomopatológico) & $\mathrm{R} \$ 57,24$ & \\
\hline Biópsia por agulhamento (incluindo anatomopatológico) & $\mathrm{R} \$ 138,36$ & \\
\hline \multirow[t]{2}{*}{ Estadiamento pré-operatório } & & Estimativa dos insumos, com os mesmos valorados \\
\hline & & pelas tabelas de remuneração do SUS \\
\hline Pacientes estadiamentos | e II * & $\mathrm{R} \$ 85,30$ & \\
\hline Pacientes estadiamento III ** & $\mathrm{R} \$ 276,29$ & \\
\hline 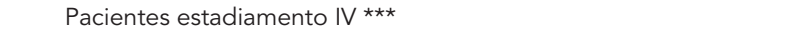 & $\mathrm{R} \$ 414,92$ & \\
\hline Tratamento cirúrgico \# & & Valores SUS \#\# \\
\hline Cirurgia conservadora & $\mathrm{R} \$ 447,49$ & \\
\hline Mastectomia & $\mathrm{R} \$ 889,23$ & \\
\hline Reconstrução mamária com prótese \#\# & $\mathrm{R} \$ 861,54$ & \\
\hline Quimioterapia (valores mensais) & & APAC \#\# \\
\hline Quimioterapia neoadjuvante & $R \$ 1.400,00$ & \\
\hline Estádio I & $\mathrm{R} \$ 571,50$ & \\
\hline Estádio II & $\mathrm{R} \$ 800,00$ & \\
\hline Estádio III & $\mathrm{R} \$ 800,00$ & \\
\hline Estádio IV (1ạ linha) & $\mathrm{R} \$ 1.700,00$ & \\
\hline Estádio IV (2a linha) & $\mathrm{R} \$ 2.378,90$ & \\
\hline $\begin{array}{l}\text { Avaliação pré-quimioterapia (consulta cardiológica e } \\
\text { ecocardiografia) }\end{array}$ & $\mathrm{R} \$ 49,94$ & \\
\hline Radioterapia & $\mathrm{R} \$ 1.138,02$ & APAC \#\# \\
\hline Tamoxifeno (valor anual) & $\mathrm{R} \$ 957,00$ & APAC \#\# \\
\hline Anastrazol (valor anual) & $\mathrm{R} \$ 3.618,00$ & APAC \#\# \\
\hline \multirow[t]{2}{*}{ Seguimento de pacientes com câncer por cinco anos (valor anual) } & & Estimativa dos insumos, com os mesmos valorados \\
\hline & & pelas tabelas de remuneração do SUS \\
\hline Pacientes estadiamentos | e || § & $\mathrm{R} \$ 194,53$ & \\
\hline Pacientes estadiamentos III e IV $\S \S$ & $\mathrm{R} \$ 385,52$ & \\
\hline Internação por intercorrência oncológica & $R \$ 860,52$ & Banco AlH §§§ \\
\hline
\end{tabular}

AlH: Autorização de Internações Hospitalares; APAC: Autorização para Procedimentos de Alta Complexidade; HCPA: Hospital de Clínicas de Porto Alegre; ICESP: Instituto do Câncer do Estado de São Paulo; INCA: Instituto Nacional de Câncer; NMPOA: Núcleo Mama Porto Alegre; SIGTAP: Sistema de Gerenciamento da Tabela Unificada de Procedimentos;

* Utilizando os seguintes insumos: ecografia transvaginal e abdominal, exames de laboratório (função renal e hepática, hemograma, glicemia), radiografia de tórax;

** Utilizando, além dos insumos dos estádios I e II, cintilografia óssea;

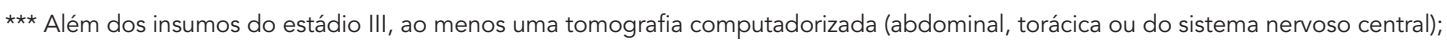

\# Baseado nas proporções por estadiamento de mastectomia radical e setorectomia descritas no texto, e nos valores das mesmas: $R \$ 889,23$ e $R \$ 447,49$, respectivamente;

\#\# Dados do Sistema de Gerenciamento da Tabela de Procedimentos, Medicamentos e OPM do SUS (SIGTAP. Departamento de Informática do SUS. http://sigtap.datasus.gov.br/, acessado em Jan/2012);

\#\#\# Realizada em 70\% das mastectomias em mulheres com menos de 70 anos e $0 \%$ nas acima desta idade; se o estadiamento for IV, não é feita a reconstrução; § Utilizando os seguintes insumos: mamografia, ecografia mamária, exames de laboratório, radiografia de tórax, ecografia abdominal e ecografia transvaginal uma vez ao ano, além de quatro consultas ao ano;

$\S \S$ Além dos insumos citados, cintilografia óssea anual;

$\S \S$ Dados do Departamento de Informática do SUS (DATASUS; http://www.datasus.gov.br). 
Dentre esses artigos, os estudos de Santa Catarina e Minas Gerais continham dados para curva de sobrevida, sendo selecionados para estimativa da sobrevida de acordo com o estadiamento inicial. Em ambos os trabalhos, a taxa de mortalidade parecia linear, sendo feita estimativa de mortalidade média anual por estádio (Tabela 1), ponderada pelo tamanho de amostra de cada estudo. Para as mulheres que não tiveram câncer no período simulado no modelo, foram usados os dados das tábuas de vida do IBGE para estimar a sobrevida de acordo com a idade.

Para o cálculo de anos de vida ajustados para qualidade, foi feita revisão da literatura a fim de levantar parâmetros de utilidade (utility). Foram encontrados cinco estudos relevantes, um deles comparando diferentes estadiamentos e os demais avaliando somente pacientes com estadiamentos precoces ou tardios 20,21,22,23,24. Após a avaliação crítica desses estudos, definiu-se os valores mostrados na Tabela 1. Consideramos que esses valores já incorporavam possíveis decrementos de qualidade de vida relacionados ao uso de quimioterapia, radioterapia e tipo de cirurgia. Para as mulheres sem câncer de mama assumiuse uma utilidade de 1 .

Estimativa dos parâmetros para mensuração dos insumos comuns a ambas as estratégias

Para a estimativa de proporção de mamografias com resultado de Breast Imaging-Reporting and Data System 3 (BI-RADS 3), optamos por utilizar a estimativa dos Parâmetros Técnicos para o Rastreamento do Câncer de Mama ${ }^{25}$, proposta pelo INCA, em que se estima que cerca de $5 \%$ das mamografias costumam apresentar um resultado de BI-RADS 3.

De acordo com as práticas clínicas correntes observadas no NMPOA e na rede pública, em termos de avaliação pré-operatória de pacientes com essa doença, os seguintes exames são feitos em todas as pacientes: ecografia abdominal e transvaginal, testes de laboratório (provas de função renal e hepática, hemograma, glicemia) e radiografia de tórax. Para as pacientes em estádios III e IV, também é preconizada a realização de cintilografia óssea, além de uma tomografia (tórax, abdômen ou crânio) para as últimas. Além disso, também foi estipulado que todas as pacientes submetidas à quimioterapia com antraciclina fariam uma ecocardiografia.

A definição do tipo de cirurgia inicial (dentre mastectomia e setorectomia) que seria realizada em cada estadiamento foi igual para ambas as estratégias. Para os estádios I a III, foram utilizados três bancos de dados (dados não publicados): o do próprio NMPOA, o do Núcleo Mama
Moinhos (serviço de mastologia com público composto por beneficiárias de planos de saúde) e o de uma coleta de dados primários feita no Hospital de Clínicas de Porto Alegre (HCPA) e no Instituto do Câncer do Estado de São Paulo (ICESP). O número total de casos nos estádios de I a III nesses três bancos era de 692, e as estimativas são baseadas em média ponderada dessas três fontes. Nos estádios I e II, as proporções de cirurgias conservadoras foram $73 \%$ e $39 \%$, respectivamente, sendo as proporções de mastectomia os valores complementares a estes. Já no estádio III, a taxa de setorectomia foi de 18\% (a maioria após quimioterapia neoadjuvante), a de mastectomia $73 \%$, havendo ainda $9 \%$ de mulheres que não realizaram nenhuma cirurgia. Para o estádio IV, foi estimado que $70 \%$ das pacientes fariam mastectomia com o intuito de redução de carga tumoral, ou alguma cirurgia do mesmo porte (o que, em termos de custos, não teria diferença significativa); as demais não teriam condições clínicas de se submeterem a qualquer procedimento cirúrgico.

Para a cirurgia de reconstrução não foram encontrados dados publicados, sendo usada a opinião de especialista. Foi estimado que, em mulheres com menos de 70 anos, $70 \%$ das que haviam sido submetidas à mastectomia nos estadiamentos de I a III fariam a reconstrução mamária com colocação de prótese; mulheres acima desta idade não fariam reconstrução. No estádio IV, foi assumido que as mulheres não fariam reconstrução.

Nesse mesmo conjunto de bancos de dados, $39 \%$ das pacientes em estádio I foram submetidas à quimioterapia. Para os estadiamentos de II a IV foi estipulado que $100 \%$ das pacientes seriam submetidas a tratamento quimioterápico; a taxa de realização de neoadjuvância no estádio II foi de $19,9 \%$, e no estádio III, $23 \%$. Tendo em vista o modelo de remuneração por quimioterapia no SUS (descrito a seguir), não definimos qual seria o esquema de terapia usado em cada uma dessas situações.

A taxa de utilização de radioterapia foi definida por opinião de especialista. Foi estipulado que, nos estadiamentos I e II, todas as pacientes submetidas à cirurgia conservadora fariam o tratamento. No estádio III, todas as pacientes seriam submetidas à radioterapia, e, no estádio IV, $50 \%$ das mesmas. Foi definido que o número de ciclos seria de 30 , no valor de $\mathrm{R} \$ 1.138,02$, segundo os valores de Autorização para Procedimentos de Alta Complexidade (APAC) do SUS (Departamento de Informática do SUS. Sistema de Gerenciamento da Tabela de Procedimentos, Medicamentos e OPM do SUS. http://sigtap.da tasus.gov.br/, acessado em Jan/2012). 
A taxa de utilização de bloqueio hormonal foi estimada com base na análise do banco de dados do Núcleo Mama Moinhos, cujo total de casos é de quase 600 pacientes. A proporção de pacientes com receptores de estrógeno ou progesterona positivos (critério para utilização de bloqueio) foi de aproximadamente $80 \%$. Estimamos que, desse total, $80 \%$ utilizarão tamoxifeno (no valor de $\mathrm{R} \$ 957,00$ ao ano), e as demais pacientes, por contraindicação clínica, usariam outro medicamento, mais comumente o anastrazol, cujo valor anual ressarcido pelo SUS é de R\$3.618,00.

No seguimento de pacientes após o diagnóstico de câncer, a estimativa de insumos utilizados teve como base as práticas correntes no atendimento de pacientes da rede pública. Anualmente, durante cinco anos, todas as pacientes fariam mamografia, ecografia mamária, abdominal e transvaginal, radiografia de tórax, além de perfil laboratorial igual ao da avaliação préoperatória (custo anual destes itens igual a $\mathrm{R} \$$ 154,53). Mulheres em estádios III e IV também fariam cintilografia óssea (R\$ 190,99). Finalmente, todas as pacientes fariam quatro consultas ao ano durante esse período (valor por consulta de $\mathrm{R} \$ 10,00)$.

A probabilidade de internações por complicações oncológicas foi estimada usando-se a análise do banco de dados do ICESP/HCPA. A frequência média de internações por ano foi de $11 \%$ no estádio I, $32 \%$ no II, $62 \%$ no III e $27 \%$ no IV. A valoração foi feita com base no valor médio de Autorização de Internações Hospitalares (AIH) para tratamento clínico de paciente oncológico, estimado em um período de 12 meses entre 2010 e 2011, no endereço eletrônico do Departamento de Informática do SUS (DATASUS; http://www.datasus.gov.br), englobando todas as AIH para este procedimento neste período no Brasil (equivalente a $\mathrm{R} \$ 860,52$ ).

Estimativa dos parâmetros para mensuração dos insumos específicos da estratégia nos moldes do NMPOA

Para a estimativa da taxa de investigação complementar com ecografia no NMPOA, foi verificada a taxa média deste exame nos últimos quatro anos, dividindo-se o total de ecografias pelo total de mamografias de rastreamento. Essa taxa foi de $23 \%$, ou seja, para cada cinco mamografias foi feita uma ecografia, aproximadamente. Durante esse mesmo período, foi estimada a taxa de complementação de mamografias de rastreamento com mamografias diagnósticas (usualmente ampliação ou compressão), que foi de 1,15\%.

Para a estimativa dos gastos com procedimentos invasivos, optamos por fazer a propor- ção entre procedimentos feitos e casos de câncer diagnosticados, a fim de obtermos um número médio de procedimentos na população rastreada para cada caso diagnosticado. No período de 2008 a 2011, foram diagnosticados 33 casos de câncer, com a realização de 22 agulhamentos, 69 biópsias de fragmento e 63 punções por agulha fina.

Finalmente, foi calculado o custo de pessoal do NMPOA (incluindo equipe médico-assistencial e administrativa), que foi igual a $\mathrm{R} \$ 54.190,00$ ao mês (já acrescido de 75\% de encargos). Levando-se em conta a capacidade assistencial máxima estimada para uma equipe com a composição atual, assim como a produtividade do mamógrafo e a frequência de rastreamento observada na população participante do projeto, estipulamos que o número total de mulheres que poderiam ser atendidas em um caráter assistencial exclusivo (sem carga horária dedicada à pesquisa, o que ocorre atualmente) seria de 9.500. Dessa forma, o custo médio por paciente para manter a estrutura de pessoal seria equivalente a $\mathrm{R} \$ 54.190,00$ * $12 / 9.500 \approx \mathrm{R} \$ 70,00$ por paciente/ano.

\section{Estimativa dos parâmetros para mensuração dos insumos específicos da estratégia cuidado usual}

Para a estimativa da utilização de mamografia no SUS foram utilizadas as seguintes informações: - De acordo com a Pesquisa Nacional por Amostra de Domicílios (PNAD) 2008, 30\% das mulheres na faixa etária dos 50-69 anos nunca realizaram mamografia 26 . Utilizamos esse valor como a proporção de mulheres que não se submeteria ao rastreamento em nenhum momento, no cuidado usual.

- Foi encontrado somente um trabalho na literatura nacional com acompanhamento prospectivo da frequência de rastreamento em usuárias do serviço público de saúde 27. Dentre as 327 mulheres que tinham realizado mamografia no início do acompanhamento, a proporção de retorno em 24 meses para nova mamografia foi de $48 \%$; somente $31,5 \%$ (do total que iniciou o estudo) retornaram em até 48 meses. Portanto, estipulamos que, em média, $40 \%$ das mulheres voltariam a cada dois anos, resultando em taxa de realização de mamografia anual no cuidado usual de $20 \%$, dentre os $70 \%$ que realizam mamografia (ou seja, $14 \%$ ao ano do total de pacientes do cuidado usual).

Não foram encontrados parâmetros para estimar a taxa de realização de biópsias, ecografias e complementos mamográficos. Para biópsias, foi utilizada a proporção de que para cada biópsia com diagnóstico de câncer, outras três 
mulheres teriam sido biopsiadas com resultado benigno. Para os outros três parâmetros, foram utilizados os mesmos valores encontrados no NMPOA (lembrando que todos são proporcionais ao número de mamografias; logo, no cuidado usual, o número absoluto de tais insumos é menor).

\section{Valoração dos insumos}

Tendo em vista que a perspectiva do presente estudo é a do SUS, todos os insumos em ambas as estratégias - NMPOA e cuidado usual - foram valorados de acordo com as tabelas do sistema, incluindo exames, procedimentos cirúrgicos e outros tratamentos (químio, rádio e hormonioterapia). A única exceção foi o custo de pessoal, no qual foram incluídos somente custos na estratégia NMPOA, com valores praticados pela Associação Hospitalar Moinhos de Vento. O motivo para não incluir custos de pessoal no cuidado usual foi porque as mulheres que fazem mamografia nesta estratégia têm a mesma solicitada por profissionais da atenção primária, os quais já seriam pagos pelo SUS da mesma forma, independentemente da atenção à saúde da mama.

Um detalhe importante na valoração dos insumos foi a não incorporação do custo de estrutura do NMPOA, tanto os gastos iniciais (aquisição de equipamentos) quando os de manutenção (incluindo aluguel de área física). Diferentemente dos valores gastos com pessoal no cuidado usual, os custos com, no mínimo, aquisição de mamógrafos, são ligados exclusivamente à saúde da mama, e seria desejável incorporá-los ao modelo. Como não foi possível estabelecer um valor para esse custo no cuidado usual (por não haver dados para custo médio por paciente dos insumos), optamos por suprimir tais custos de ambas as estratégias.

\section{Análises de sensibilidade}

Análises de sensibilidade univariadas (one-way) foram conduzidas nos principais parâmetros do modelo, com especial atenção para aqueles com estimativas frágeis. Os parâmetros incluídos, e suas respectivas variações, foram os seguintes:

- Taxa de desconto, variando entre $0 \%$ e $10 \%$;

- Taxa de mamografias com resultado de BIRADS 3, entre $1 \%$ e $10 \%$;

- Custo de pessoal do NMPOA, entre $-50 \%$ e $+100 \%$ por paciente por ano;

- Taxa de realização de ecografia, com redução de $50 \%$ em ambas as estratégias;

- Incidência de câncer de mama, com redução de $20 \%$;
- Adesão das participantes ao rastreamento na estratégia nos moldes do NMPOA, com aumento de $10 \%$;

- Melhora dos estadiamentos do cuidado usual, refletindo o dado com maior taxa de estadiamentos precoces da literatura nacional 16;

- Valores de utilidade, oscilando para mais ou menos $20 \%$.

Tendo em vista que a maioria das decisões de oscilação de parâmetros descritas anteriormente foi arbitrária, isto é, não baseada em uma distribuição de probabilidades efetivamente observada no NMPOA, optamos por não realizar análise de sensibilidade probabilística, uma vez que a decisão sobre tipo e parametrização de distribuições na mesma teria limitações importantes.

\section{Resultados}

A expectativa média de vida ajustada para qualidade para mulheres entre 40-69 anos (idade média da coorte hipotética igual a 53 anos) no cuidado usual foi de 13,97 QALY (ou 27,59 QALY, sem aplicação da taxa de desconto). Na estratégia de rastreamento nos moldes do NMPOA, a expectativa de vida foi de 14,05 QALY (ou 27,86 QALY, sem desconto). O custo cumulativo médio no cuidado usual foi de $\mathrm{R} \$ 1.008,00$, e na estratégia NMPOA, de R $\$ 2.115,00$. A relação de custo-efetividade incremental foi de $\mathrm{R} \$ 13.426,00$ por QALY na comparação entre as estratégias. A relação de custo-efetividade incremental não descontada seria de R \$ 9.007,00 por QALY. Esses valores são mostrados na Tabela 2, que também apresenta os resultados em anos de vida ganhos (sem ajuste para qualidade).

\section{Validação do modelo}

Não foi possível validar prospectivamente o modelo desenvolvido como um todo, pela ausência de coortes brasileiras com dados de incidência e mortalidade por câncer de mama. Medidas indiretas, como comparações com outros modelos internacionais e dados de sobrevida do Brasil, permitem uma análise parcial. Por exemplo, com base nas tábuas de vida do IBGE para a população brasileira com a distribuição etária assumida no modelo, a expectativa de vida seria de 28,5 anos, valor próximo ao efetivamente encontrado no modelo na estratégia cuidado usual, em que a expectativa de vida foi de 27,84 anos.

Também comparamos, com fins de validação, a proporção dos estadiamentos estimados pelo modelo com os efetivamente observados no NMPOA até o momento. De acordo com o modelo, a proporção seria de 52,3\% no estádio I, 31,2\% 
Custo, efetividade e relação de custo-efetividade incremental entre as estratégias em comparação.

\begin{tabular}{lccccc} 
& Custo total (R\$) & \multicolumn{2}{c}{ Efetividade } & \multicolumn{2}{c}{ Custo-efetividade incremental } \\
& & Anos de vida & QALY & R\$/Anos de vida & R\$QALY \\
\hline Cuidado usual & $1.008,00$ & 14,05 & 13,97 & - & - \\
NMPOA & $2.115,00$ & 14,11 & 14,05 & 18,667 & $13.426,00$ \\
\hline
\end{tabular}

NMPOA: Núcleo Mama Porto Alegre; QALY: quality adjusted life years.

Nota: Todos os valores apresentados foram submetidos à taxa de desconto de $5 \%$ ao ano.

no II, $11,4 \%$ no III e 5,1\% no IV. Na casuística efetivamente observada, a distribuição foi de $52,5 \%$ no estádio I, 36,1\% no II, 9,8\% no III e 1,6\% no IV.

\section{Análises de sensibilidade}

Todas as análises de sensibilidade realizadas são mostradas na Tabela 3. Os valores observados não diferem marcadamente em relação ao caso base. Um incremento relativo de $10 \%$ na adesão (anual, bianual e trianual) do programa de rastreamento nos moldes do NMPOA tornaria a relação de custo-efetividade incremental levemente mais favorável (R $\$ 12.515,00 / \mathrm{QALY})$. Modificando-se os estadiamentos do rastreamento no cuidado usual para refletir detecção mais precoce, semelhante ao estudo gaúcho 16 (estádio I, 20\%; estádio II, 58\%; estádio III, 15\%; e estádio IV, 7\%), a relação de custo-efetividade incremental elevaria para $\mathrm{R} \$ 22.511,00 / \mathrm{QALY}$, o que ainda é atrativa do ponto de vista econômico.

A incidência de câncer de mama modelada refletiu aquela da população de Porto Alegre, considerada alta pela literatura, estando, juntamente com a cidade do Rio de Janeiro, entre as mais altas do Brasil. No cenário em que a incidência fosse $20 \%$ menor, a relação de custoefetividade incremental seria elevada para $\mathrm{R} \$$ 17.178,00/QALY.

Variando os custos de pessoal com o programa de rastreamento nos moldes do NMPOA em $-50 \%$ a $+100 \%$, os resultados da relação de custo-efetividade incremental variariam entre R\$ 7.432,00 e R\$ 25.413,00/QALY. Com taxas de desconto entre $0 \%$ e $10 \%$, a relação de custo-efetividade incremental oscilaria entre R\$9.007,00 e $\mathrm{R} \$ 18.441,00 / \mathrm{QALY}$.

\section{Discussão}

Neste trabalho, em que se objetivou estimar a relação de custo-efetividade incremental de um programa de rastreamento centralizado, com proposta de mamografia e exame clínico anuais para mulheres entre 40 e 69 anos de idade, foi vista uma relação de custo-efetividade aceitável do ponto de vista econômico de cerca de R\$ 13.000,00 por QALY. Essa estimativa está situada abaixo do valor equivalente a uma vez o PIB per capita do Brasil (ano de 2010), valor considerado bastante atrativo do ponto de vista farmacoeconômico ${ }^{28}$. Nas análises de sensibilidade realizadas, a maioria dos cenários não teve alterações significativas (considerando como referência o limiar de uma vez o PIB per capita), com algumas situações em que a relação de custo-efetividade incremental esteve situada entre uma e três vezes o PIB per capita (valor ainda interessante sob o prisma econômico), como no cenário de aumento em $100 \%$ do custo de pessoal da estratégia nos moldes do NMPOA.

Até o momento, havia apenas um estudo que tinha realizado a avaliação econômica do rastreamento mamográfico em cenário nacional, porém comparando cenários pouco verossímeis - um com $100 \%$ e outro com $0 \%$ de realização de mamografia 9. A maioria dos trabalhos internacionais também utilizou apenas esses cenários 7,29,30. Em um dos poucos estudos na literatura que comparou a realidade atual em termos de rastreamento de câncer de mama com outras opções, nos Estados Unidos, foi visto que os custos para rastrear mulheres entre 50 e 75 anos de idades a cada dois anos eram menores que os gastos incorridos com rastreamento naquele momento no país, apresentando uma efetividade maior, desta forma dominando o cenário corrente de rastreamento ${ }^{6}$. Alguns cenários alternativos, como rastreamento dos 45 aos 75 anos a cada dois anos, eram extremamente atrativos do ponto de visto econômico na comparação com os padrões atuais de rastreamento, com uma relação de custo-efetividade incremental de apenas US\$ 8.000,00 por QALY. Rastrear essa mesma faixa etária com intervalo anual, por outro lado, apre- 
Variação dos parâmetros na análise de sensibilidade e relações de custo-efetividade incrementais resultantes.

\begin{tabular}{lcc}
\hline Parâmetros & Oscilação & $\begin{array}{c}\text { Variação na relação de custo- } \\
\text { efetividade incremental (R\$/QALY) }\end{array}$ \\
\hline Taxa de desconto & $0 \% ; 10 \%$ & $9.007,00 ; 18.441,00$ \\
Taxa de mamografias com BIRADS 3 & $1 \% ; 10 \%$ & $13.306,00 ; 13.576,00$ \\
Custo de pessoal do NMPOA & $-50 \% ;+100 \%$ & $7.432,00 ; 25.413,00$ \\
Taxa de realização de ecografia em ambas as & $-50 \%$ & $13.242,00$ \\
estratégias & $-20 \%$ & $17.178,00$ \\
Incidência de câncer de mama & $+10 \%$ & $12.515,00$ \\
Adesão das participantes ao rastreamento (NMPOA) & $*$ & $22.511,00$ \\
Melhora dos estadiamentos do cuidado usual & $-20 \% ;+20 \%$ & $16.782,00 ; 11.188,00$ \\
Valores de utilidade & & \\
\hline
\end{tabular}

NMPOA: Núcleo Mama Porto Alegre; QALY: quality adjusted life years.

* Casuística de Santa Maria (Rio Grande do Sul), publicação nacional com estadiamentos mais favoráveis 16.

sentou uma relação de custo-efetividade incremental um pouco menos atrativa, de US $\$ 35.000$ por QALY. É importante mencionar que, nesse trabalho, os cenários alternativos consideravam $100 \%$ de adesão ao esquema proposto, o que é pouco verossímil. No nosso trabalho, utilizamos dados reais de adesão observados em um programa de rastreamento conduzido em cenário nacional.

\section{Limitações}

O presente estudo apresenta algumas limitações. Primeiramente, seus resultados são fruto dos parâmetros estimados para o modelo, os quais nem sempre foram baseados em informações locais - alguns tiveram por base dados internacionais, e, quando estes também eram ausentes, as estimativas foram feitas com a opinião de especialista. Dessa forma, é importante que se leve em consideração a fragilidade de alguns parâmetros na interpretação dos achados.

Em segundo lugar, tendo em vista o curto tempo de seguimento da coorte do NMPOA e o pequeno número de casos de câncer detectados até o momento, optamos por estimar os efeitos deste programa de rastreamento de forma indireta, relacionando a adesão ao rastreamento com estadiamentos dos casos de câncer. Por outro lado, os resultados estimados são semelhantes à casuística efetivamente observada. Além disso, o objetivo deste trabalho era estimar o custoefetividade de um programa de rastreamento semelhante ao NMPOA aplicado à população em geral de mulheres usuárias do SUS; entretanto, a população do NMPOA é provavelmente um pouco mais aderente ao rastreamento, uma vez que a mesma aceitou o convite para participar do projeto. Dessa forma, é possível que a relação de custo-efetividade incremental fosse um pouco menos favorável em uma população não selecionada.

Outra limitação do modelo foi a impossibilidade, por detalhes técnicos, de fazer com que as pacientes tivessem progressão ao longo do modelo. Tal fato não tem implicação na sobrevida, uma vez que as estimativas desta de acordo com o estadiamento inicial já levavam em conta a progressão da doença; porém, custos observados em pacientes que progridem, como novos gastos com quimioterapia, não foram computados. Dessa maneira, o substancial número de casos em estádios II e III do cuidado usual provavelmente teria maior custo associado do que foi de fato incorporado no modelo, o que faria com que a relação de custo-efetividade incremental se mostrasse mais favorável à estratégia nos moldes do NMPOA caso estes valores pudessem ser contabilizados.

Também é importante lembrar que não foram incorporados custos de estrutura em ambas as estratégias, por problemas já citados na seção de métodos. Porém, mesmo considerando um valor de pessoal dobrado no NMPOA (o que provavelmente compensaria esta cifra, uma vez que o incremento utilizado na análise de sensibilidade foi de $100 \%$, incorrendo em um incremento de $\mathrm{R} \$ 650.000,00$ anuais para uma população rastreada de 9.500 mulheres), a relação de custo-efetividade incremental permaneceu dentro de limites aceitáveis, em cerca 
de R\$ 25.000,00 por QALY. Por outro lado, não foram incorporados custos de estrutura do SUS, de modo que a relação de custo-efetividade incremental mais realista provavelmente seria um valor intermediário entre este e o observado no caso base.

Outros parâmetros com dificuldade de estimativa foram os estadiamentos que seriam observados nas pacientes em rastreamento irregular ou sem rastreamento no NMPOA, e taxas de ecografia e mamografia complementares no cuidado usual. A direção dos resultados com os parâmetros mais acurados é pouco previsível. Também digno de nota é a utilização de valores remunerados pelo SUS, os quais não correspondem, necessariamente, aos custos reais dos insumos. Porém, tendo em vista que a perspectiva do projeto era a do SUS, esses valores eram os mais corretos para esse fim.

Finalmente, também é importante salientar que a análise feita neste trabalho englobou pacientes entre 40 e 69 anos, sem uma divisão por faixa etária, sendo a opção mais interessante a comparação entre a inclusão ou não de mulheres de 40 a 49 anos, em que o custo-efetividade parece ser pior, conforme trabalhos internacionais 31 .

\section{Considerações finais}

O câncer de mama apresenta alta incidência em várias regiões do país, tendendo a aumentar, tendo em vista o envelhecimento da população. Dentre as estratégias para diminuir a morbimortalidade associada, a mais bem estabelecida é o rastreamento mamográfico. Neste trabalho, foi visto que um programa organizado de rastreamento apresenta custo-efetividade aceitável, apesar da ressalva quanto à imprecisão de alguns parâmetros utilizados no modelo econômico. A melhor apropriação de dados epidemiológicos nacionais é fundamental para aumentar a capacidade preditiva do modelo construído.

\section{Resumen}

El objetivo de este estudio fue evaluar el coste-efectividad de un programa organizado para el diagnóstico precoz con mamografía del cáncer de mama, implementado en Porto Alegre, Rio Grande do Sul, Brasil (Núcleo Mama Porto Alegre-NMPOA). Se utilizó el modelo de Markov, con el fin de estimar la tasa de costeefectividad incremental de NMPOA, comparada con la situación actual en lo referente a la atención del cáncer de mama en el sistema público de salud brasileño. El estudio se realizó en una cohorte hipotética de mujeres entre 40-69 años con riesgo de cáncer de mama. Los parámetros se obtuvieron del NMPOA y literatura nacional. En la estrategia del NMPOA, la eficacia se modeló teniendo en cuenta la adhesión real de la población al programa. La efectividad se midió en años de vida ajustados por calidad (QALY). La tasa de coste-efectividad incremental en el caso base fue $R \$ 13.426,00$ por QALY. Este resultado no fue sensible a la variación de los principales parámetros del modelo en el análisis de sensibilidad. Teniendo en cuenta el umbral frecuentemente aceptado como muy alto económicamente en Brasil, un programa similar al NMPOA es coste-efectivo en ciudades con alta incidencia de cáncer de mama.

Neoplasias de la Mama; Tamizaje Masivo; Costo Efectividad; Evaluación de Tecnologías de Salud

\section{Colaboradores}

R. A. Ribeiro participou da concepção do modelo econômico, realização das análises e redação do artigo. M. Caleffi colaborou na revisão do artigo final e construção do modelo. C. A. Polanczyk contribuiu na concepção do modelo, interpretação dos dados e revisão do artigo final.

\section{Agradecimentos}

Agradecemos a toda equipe envolvida no atendimento às pacientes e coleta de dados no Núcleo Mama Porto Alegre. Pesquisa realizada no âmbito do Projeto Núcleo Mama Porto Alegre - Desenvolvimento de Técnicas de Operação e Gestão de um Serviço de Atenção à Saúde da Mama, de acordo com o Programa de Apoio ao Desenvolvimento Institucional do Sistema Único (PROADI-SUS), firmado entre o Ministério da Saúde e a Associação Hospitalar Moinhos de Vento, por meio do termo de ajuste de número 06/2008, assinado em 17 de novembro de 2008

\section{Conflito de interesses}

A coautora Maira Caleffi é presidente da Federação Brasileira de Instituições Filantrópicas de Apoio à Saúde da Mama (FEMAMA). 


\section{Referências}

1. Instituto Nacional de Câncer. Estimativa 2012: incidência de câncer no Brasil. Rio de Janeiro: Instituto Nacional de Câncer; 2011.

2. Kemp C, Elias S, Gebrim L, Nazario A, Baracat E, Lima G. Estimativa de custo do rastreamento mamográfico em mulheres no climatério. Rev Bras Ginceol Obstet 2005; 27:415-20.

3. Duffy SW, Tabar L, Chen HH, Holmqvist M, Yen MF, Abdsalah S, et al. The impact of organized mammography service screening on breast carcinoma mortality in seven Swedish counties. Cancer 2002; 95:458-69.

4. Humphrey LL, Helfand M, Chan BK, Woolf SH. Breast cancer screening: a summary of the evidence for the U.S. Preventive Services Task Force. Ann Intern Med 2002; 137(5 Part 1):347-60.

5. Instituto Nacional de Câncer, Ministério da Saúde. Controle do câncer de mama: documento de consenso. Rev Bras Cancerol 2004; 50:77-90.

6. Stout NK, Rosenberg MA, Trentham-Dietz A, Smith MA, Robinson SM, Fryback DG. Retrospective cost-effectiveness analysis of screening mammography. J Natl Cancer Inst 2006; 98:774-82.

7. Wong IO, Kuntz KM, Cowling BJ, Lam CL, Leung GM. Cost effectiveness of mammography screening for Chinese women. Cancer 2007; 110:885-95.

8. Carles M, Vilaprinyo E, Cots F, Gregori A, Pla R, Roman R, et al. Cost-effectiveness of early detection of breast cancer in Catalonia (Spain). BMC Cancer 2011; 11:192.

9. Peregrino AA, Vianna CM, Almeida CE, Gonzáles GB, Machado SC, Silva FVC, et al. Análise de custo-efetividade do rastreamento do câncer de mama com mamografia convencional, digital e ressonância. Ciênc Saúde Colet 2012; 17:215-22.

10. Caleffi M, Ribeiro RA, Duarte Filho DL, AshtonProlla P, Bedin Jr. AJ, Skonieski GP, et al. A model to optimize public health care and downstage breast cancer in limited-resource populations in southern Brazil. (Porto Alegre Breast Health Intervention Cohort). BMC Public Health 2009; 9:83.

11. Tabar L, Duffy SW, Vitak B, Chen HH, Prevost TC. The natural history of breast carcinoma: what have we learned from screening? Cancer 1999; 86: 449-62.

12. Schneider IJ, d'Orsi E. Sobrevida em cinco anos e fatores prognósticos em mulheres com câncer de mama em Santa Catarina, Brasil. Cad Saúde Pública 2009; 25:1285-96.

13. Guerra MR, Mendonça GA, Bustamante-Teixeira MT, Cintra JR, Carvalho LM, Magalhães LM. Sobrevida de cinco anos e fatores prognósticos em coorte de pacientes com câncer de mama assistidas em Juiz de Fora, Minas Gerais, Brasil. Cad Saúde Pública 2009; 25:2455-66.

14. Thuler LCS, Mendonça GA. Initial staging of breast and cervical cancer in Brazilian women. Rev Bras Ginecol Obstet 2005; 27:656-60.
15. Brito C, Portela MC, Vasconcellos MT. Survival of breast cancer women in the state of Rio de Janeiro, Southeastern Brazil. Rev Saúde Pública 2009; 43:481-9.

16. Moraes AB, Zanini RR, Turchiello MS, Riboldi J, Medeiros LR. Estudo da sobrevida de pacientes com câncer de mama atendidas no hospital da Universidade Federal de Santa Maria, Rio Grande do Sul, Brasil. Cad Saúde Pública 2006; 22:2219-28.

17. White E, Miglioretti DL, Yankaskas BC, Geller BM, Rosenberg RD, Kerlikowske K, et al. Biennial versus annual mammography and the risk of late-stage breast cancer. J Natl Cancer Inst 2004; 96:1832-9.

18. Instituto Nacional de Câncer. Câncer no Brasil: dados dos registros de base populacional. v. IV. Rio de Janeiro: Instituto Nacional de Câncer; 2010.

19. Breast Screening Frequency Trial Group. The frequency of breast cancer screening: results from the UKCCCR Randomised Trial. United Kingdom Co-ordinating Committee on Cancer Research. Eur J Cancer 2002; 38:1458-64.

20. Schleinitz MD, DePalo D, Blume J, Stein M. Can differences in breast cancer utilities explain disparities in breast cancer care? J Gen Intern Med 2006; 21:1253-60.

21. Lloyd A, Nafees B, Narewska J, Dewilde S, Watkins J. Health state utilities for metastatic breast cancer. Br J Cancer 2006; 95:683-90.

22. Garrison Jr. LP, Lubeck D, Lalla D, Paton V, Dueck A, Perez EA. Cost-effectiveness analysis of trastuzumab in the adjuvant setting for treatment of HER2positive breast cancer. Cancer 2007; 110:489-98.

23. Millar JA, Millward MJ. Cost effectiveness of trastuzumab in the adjuvant treatment of early breast cancer: a lifetime model. Pharmacoeconomics 2007; 25:429-42.

24. Martin SC, Gagnon DD, Zhang L, Bokemeyer C, van Marwijk Kooy M, van Hout B. Cost-utility analysis of survival with epoetin-alfa versus placebo in stage IV breast cancer. Pharmacoeconomics 2003; 21:1153-69.

25. Instituto Nacional de Câncer. Parâmetros técnicos para o rastreamento do câncer de mama. Rio de Janeiro: Instituto Nacional de Câncer; 2009.

26. Instituto Brasileiro de Geografia e Estatística. Pesquisa Nacional por Amostra de Domicílios: acesso e utilização de serviços de saúde. Rio de Janeiro: Instituto Nacional de Câncer; 2008.

27. Marchi AA, Gurgel MSC. Adesão ao rastreamento mamográfico oportunístico em serviços de saúde públicos e privados. Rev Bras Ginecol Obstet 2010; 32:191-7.

28. WHO Commission on Macroeconomics and Health. Macroeconomics and health: investing in health for economic development. Geneva: World Health Organization; 2001. 
29. Rosenquist CJ, Lindfors KK. Screening mammography beginning at age 40 years: a reappraisal of cost-effectiveness. Cancer 1998; 82:2235-40.

30. Okonkwo QL, Draisma G, der Kinderen A, Brown ML, de Koning HJ. Breast cancer screening policies in developing countries: a cost-effectiveness analysis for India. J Natl Cancer Inst 2008; 100:1290-300.
31. Mandelblatt JS, Cronin KA, Bailey S, Berry DA, de Koning HJ, Draisma G, et al. Effects of mammography screening under different screening schedules: model estimates of potential benefits and harms. Ann Intern Med 2009; 151:738-47.

Recebido em 16/Abr/2013

Versão final reapresentada em 24/Jul/2013

Aprovado em 30/Jul/2013 